\title{
Defect Dynamics in Artificial Colloidal Ice: Real-Time Observation, Manipulation, and Logic Gate
}

\author{
Johannes Loehr, ${ }^{1,2}$ Antonio Ortiz-Ambriz, ${ }^{1,3}$ and Pietro Tierno ${ }^{1,3, *}$ \\ ${ }^{1}$ Departament de Física de la Matèria Condensada, Universitat de Barcelona, Barcelona 08028, Spain \\ ${ }^{2}$ Physikalisches Institut, Universität Bayreuth, 95440 Bayreuth, Germany \\ ${ }^{3}$ Institut de Nanociència i Nanotecnologia, IN ${ }^{2} U B$, Universitat de Barcelona, Barcelona 08028, Spain \\ (Received 2 June 2016; revised manuscript received 16 August 2016; published 12 October 2016)
}

\begin{abstract}
We study the defect dynamics in a colloidal spin ice system realized by filling a square lattice of topographic double well islands with repulsively interacting magnetic colloids. We focus on the contraction of defects in the ground state, and contraction or expansion in a metastable biased state. Combining realtime experiments with simulations, we prove that these defects behave like emergent topological monopoles obeying a Coulomb law with an additional line tension. We further show how to realize a completely resettable "NOR" gate, which provides guidelines for fabrication of nanoscale logic devices based on the motion of topological magnetic monopoles.
\end{abstract}

DOI: 10.1103/PhysRevLett.117.168001

Geometric frustration is a complex phenomenon which encompasses a broad range of systems, from magnetic materials [1], to ferroelectrics [2], trapped ions [3], confined microgel particles [4], and folding proteins [5]. It emerges when the spatial arrangement of the system elements cannot simultaneously minimize all interaction energies, and leads to exotic phases of matter with a low-temperature degenerate ground state, such as spin ice [6-8]. Artificial spin ice systems (ASI) are lattices of interacting nanoscale ferromagnetic islands, recently introduced as a versatile model to investigate geometrically frustrated states $[9,10]$, including the role of disorder $[11,12]$, thermalization [13-15], and the excitation dynamics [16-20]. In opposition to bulk spin ice such as pyrochlore compounds, ASI allow us to directly visualize the spin textures and to tailor the spatial arrangement of the system elements.

An intriguing aspect in ASI, which is attracting much theoretical interest, is the dynamics of defects [21-28]. The interactions between pairs of defects is one of the distinctive features between three dimensional (3D) and two dimensional (2D) spin ice. In a 3D pyrochlore compound, the spins are located on a lattice of corner-sharing tetrahedra, and can point either towards the tetrahedra center (spin in), or away from it (spin out). Thus the ground state (GS) follows the "ice rules," with two spins coming in and two going out of each vertex in order to decrease the vertex energy. At finite temperature, defects that behave like "magnetic monopoles" [29,30] can emerge when a spin flips, producing a local increase of the magnetic energy. A way to overcome the system complexity is to use the "dumbbell" model [31], which only considers the magnetic charge distribution at the vertices of the lattice. Within this formalism, it was shown that in 3D spin ice, a pair of defects connected by strings of flipped spins only interact through a magnetic Coulomb law at low temperature. In contrast, numerical simulations show that for a 2D square ASI, i.e., a projection of the 3D ice system on a plane, such a string requires an additional energetic term in the form of a line tension [21]. The reason is that, while in a 3D system all spin configurations that satisfy the ice rules have equal energy, in the 2D square ASI the distance at a vertex between opposing spins is greater than the distance between adjacent spins. This results in a lift of the degeneracy of the ground state, which is now represented by a twofold degenerate antiferromagnetic order.

String tension and the Coulombic interactions in ASI have been calculated by Monte Carlo simulations [22,23,27]; however, direct experimental measurements remain elusive. The difficulty of preparing the system in the GS and the extremely fast spin dynamics in nanoscale ASI makes real-time observation challenging, suggesting the use of alternative systems. Here we overcome these limitations by realizing an artificial colloidal spin ice system, a microscale soft matter analog of a frustrated nanoscale ASI. In this system we investigate the real-time dynamics of monopolelike defects via experiments and numerical simulations, and directly measure the line tension and Coulombic contributions. Further, we demonstrate defect manipulation via external field, and realize a logic operation based on magnetic current.

Our experimental system is inspired by previous theoretical works on electrostatically interacting colloids in bistable optical traps [32,33]. The schematic in Fig. 1(a) and the experimental realization in Fig. 1(b) illustrate the main idea. By soft lithography, we realize a square lattice of bistable topographic traps with lattice constant $a=29 \mu \mathrm{m}$. Each trap is composed of two wells of depth $\sim 3 \mu \mathrm{m}$, connected by a small hill at the middle with average elevation $\langle h\rangle=0.86 \mu \mathrm{m}$, Figs. 1(d)-1(f) [34]. These traps 

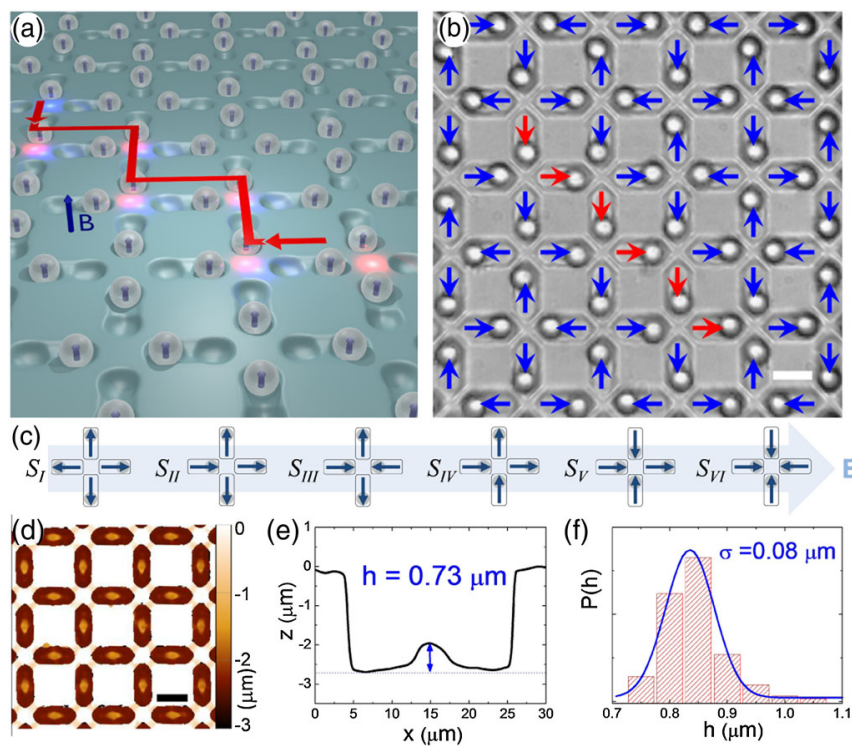

FIG. 1. (a) Schematic showing the colloidal spin ice composed by interacting colloids in a square lattice of double wells. The red line shows a defect line separating two $q= \pm 2$ defects in the GS. (b) Microscope image of an experimental defect line in a square lattice of lithographic double wells filled with paramagnetic colloids. Blue arrows denote spin directions, red arrows highlight the defect line. Scale bar is $15 \mu \mathrm{m}$. (c) Vertex configurations for the colloidal square ice. Vertex energy increases from left to right. (d) Optical profilometer image of the lithographic square lattice. (e) Cross section of a typical double well characterized by a central hill of height $h=0.73 \mu \mathrm{m}$. (f) Distribution of hill height $h$ fitted with a Gaussian function (continuous line).

are designed to confine a colloidal particle in one of the two sides, such that the particle can cross the hill when subjected to an external force, but it cannot escape from the bistable confinement. We induce repulsive interactions by using paramagnetic colloids with diameter $d=$ $10.3 \mu \mathrm{m}$ and magnetic volume susceptibility $\chi=0.08$ (Microparticles $\mathrm{GmbH}$ ). Under an external magnetic field perpendicular to the particle plane, $\boldsymbol{B}=B_{z} \hat{z}$, the colloids repel by a tunable pair potential, $U_{i j}^{m}=\omega\left(a^{3} / r_{i j}^{3}\right)$, where $\omega=\mu_{0} m^{2} /\left(4 \pi a^{3}\right)$ is the coupling constant with $\boldsymbol{m}=$ $\pi d^{3} \chi \boldsymbol{B} /\left(6 \mu_{0}\right)$ the induced moment, $r_{i j}=\left|\boldsymbol{r}_{i}-\boldsymbol{r}_{j}\right|$, and $r_{i}$ is the position of particle $i$. The gravitational potential for a particle to jump a hill is $U_{g}^{\text {hill }}=910 k_{B} T$, and $U_{g}^{\text {wall }}=$ $3740 k_{B} T$ to leave the bistable trap. Here $k_{B}$ is the Boltzmann constant, $T=293 \mathrm{~K}$, and we apply the external field such that $U_{g}^{\text {hill }}<U^{m}<U_{g}^{\text {out }}$ [37].

Once filled with one particle per double well, one can assign a vector (analogous to a spin) to each particle, such that it points from the free well to the well occupied by the particle. As shown in Fig. 1(c), it is possible to construct a set of ice rules for the colloidal artificial ice similar to the nanoscale ASI [32,38]. Vertices with three $\left(S_{V}\right)$ or four $\left(S_{V I}\right)$ colloids in are energetically unfavorable, and they are topologically connected with low energy vertices having three $\left(S_{I I}\right)$ or four $\left(S_{I}\right)$ colloids out. Thus, the GS is composed of $S_{I I I}$ vertices [35], while the metastable biased state has high energy $S_{I V}$ vertices. Both configurations satisfy the ice rules. According to the dumbbell model [31], we can associate to each spin a "magnetic charge," which is positive (negative) for spin in (out). The total charge at each vertex $i$ is given by the sum over all neighboring spins $q=\sum_{i} q_{i}$, and both the GS and the biased state correspond to $q=0$, while all other vertices have a net charge.

We start by analyzing the contraction of a pair of $q= \pm 2\left(S_{I I}\right.$ and $\left.S_{V}\right)$ charged defects connected by a line of six flipped spins along the diagonal in the GS, Figs. 1(b) and 2(a) [39]. After preparing the system with the optical tweezers, we switch the field on and measure the relaxation toward equilibrium. As shown in Fig. 2(a) and VideoS1 in Ref. [34], both defects approach via a stepwise flipping of the colloids position and the system recovers the GS. Theoretical work [22] based on the dumbbell model [31] predicts the interaction potential between the two defects in the 2D ASI as $V(l)=-Q / l+\kappa l+c$. Here, $Q$ is the topological Coulombic charge, $\kappa$ the line tension, and $c$ a constant associated with the creation of the defect pairs [25]. We confirm the validity of this assumption in our system, by explicitly calculating the energy cost $V(l)=$ $E_{\text {exc }}(l)-E_{\mathrm{GS}}$ of a defect line of length $l$ in the GS, which can be obtained by subtracting the GS energy from the energy of the excited configuration. The magnetic energy is given by the sum of all dipole interactions as $E=\sum_{i} \sum_{j \neq i} U_{i j}^{m}$. In the inset of Fig. 2(b), we show the normalized potential $V(l) / \omega$. We subtract its linear part in order to emphasize the presence of a magnetic Coulombic term. Since $V(l)$ scales with the coupling constant, it follows that $Q, \kappa \sim \omega \sim H^{2}$. By fitting this potential, we obtain the ratio $Q / \kappa=0.0290 \pm 0.0014 a^{2}$ between the Coulombic and line tension contribution, which is 1 order of magnitude lower than the corresponding one found for ASI [21].

Figure 2(b) shows experiments and simulations of the average line length $\langle l\rangle$ obtained by measuring the particle residence time within the traps [34]. We describe the dynamics of the defect line with an overdamped equation of motion with a friction coefficient $\gamma$ :

$$
\gamma \frac{d l}{d t}=-\frac{\partial V}{\partial l}=-\frac{Q}{l^{2}}-\kappa .
$$

We assume negligible the thermal fluctuations given the large size of the employed particles, and we justify our choice of overdamped dynamics, as opposed to the infradamped dynamics in nanoscale ASI [27], by checking that the defect motion effectively shows a velocity profile linear with the applied force [34]. By solving Eq. (1), we fit its solution to the experimental data in Fig. 2(b) [34]. We use the ratio $Q / \kappa$ obtained from the calculation of $V(r)$ [inset 

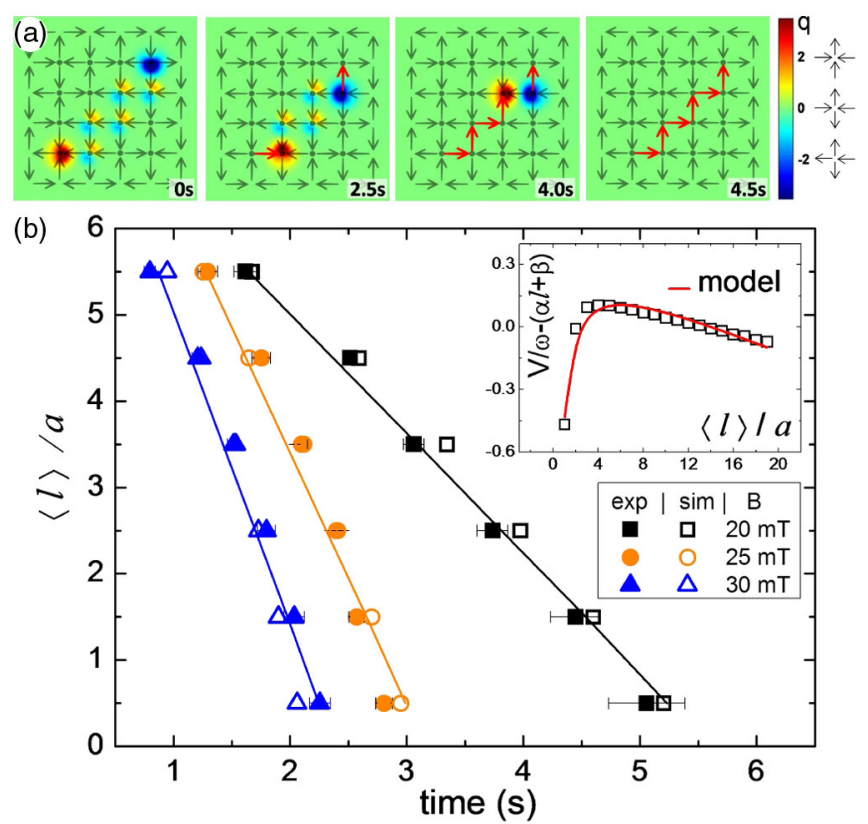

FIG. 2. (a) Color map showing the net vertex charges in the experiments for a defect line connecting two $q= \pm 2$ defects under a field $B_{z}=25.7 \mathrm{mT}$ (VideoS1 in Ref. [34]). The line consists of high energy $S_{I V}$ vertices with a zero charge but a net dipole, which give raise to the additional line-tension term. (b) Average line length $\langle l\rangle$ versus time for three different magnetic fields. Closed (empty) symbols denote experiments (numerical simulation), continuous lines are fit from Eq. (1) in the text. Inset: normalized interaction potential $V(l) / \omega$ between two topological defects minus its linear contribution $(\alpha l+\beta)$. Red line is a fit using the potential described in the text.

Fig. 2(b)], and reduce to $Q / \gamma$ the sole unknown parameter. Figure 2(b) shows the results of this procedure, confirming that the observed phenomena are well captured by Eq. (1). In all our analysis we use $\gamma$ as the scaling factor for the topological Coulomb charge $Q$. However, $Q$ may be estimated in first approximation by considering that the defects are composed by colloidal particles approaching at a constant speed in a liquid medium [40]. For an applied field of $B_{z}=30 \mathrm{mT}$, we obtain for the colloidal spin ice $Q_{M} \sim \sqrt{4 \pi|Q| / \mu_{0}}=5.7 \pm 1.5 \times 10^{-8} \mathrm{~m} / \mathrm{s}$. To further validate our analysis, we complement the experimental measurements with Brownian dynamics simulation, following the scheme described in Ref. [34]. In the simulation, we use the same experimental parameters and disorder level, and find again very good agreement with the measured data, Fig. 2(b).

We can clearly visualize the effect of the magnetic Coulombic contribution by studying defect motion in the biased system, which can be prepared by displacing all particles towards one of the system corners with the optical tweezers. In this state, it is possible to generate defect lines characterized by positive or negative line tension, or single defects with zero Coulombic contribution that propagate
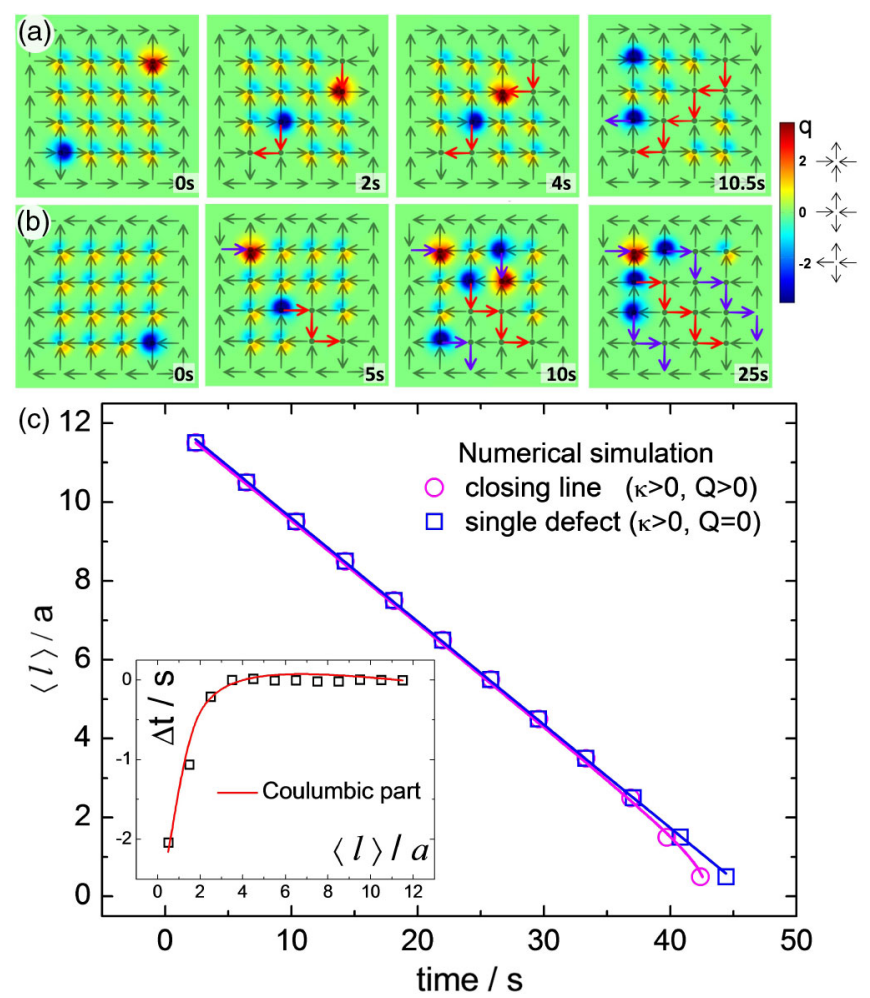

FIG. 3. (a),(b) Experimental vertex charges for a closing line (a) and for a single $q=-2$ propagating defect (b) in the biased state. Black (blue) arrows are spins flipped by the motion of the original (spontaneously emerged) defects. Corresponding movies (VideoS2, VideoS3) are in Ref. [34]. (c) Numerical simulation of case (a) (with one end fixed) and (b) showing the evolution of the line length $\langle l\rangle$ for an applied field $B_{z}=18.8 \mathrm{mT}$. Continuous lines are fits from Eq. (1). Bottom inset: difference between the two curves in the main panel (empty squares) versus line length plotted with Eq. (1) with $\kappa=0$ (continuous line).

along a diagonal [41]. Of these three cases, Figs. 3(a) and 3(b) show the first and the last one; the rest is in Ref. [34]. The first case is shown in Fig. 3(a), where two $q= \pm 2$ defects approach when an external field $B_{z}=$ $25.7 \mathrm{mT}$ is applied, leaving a series of $S_{I I I}$ vertices behind. This situation is similar to the defect motion in the GS, with attractive line tension and Coulombic interaction. We also calculate the interaction potential $V(l)$ (data not shown), obtaining an almost identical plot as the inset in Fig. 2(c). In contrast, in Fig. 3(b) a single $q=-2$ defect propagates along the lattice only due to line tension, since the absence of other charges sets the Coulombic term in Eq. (1) to 0. In the bias state we find that the defect dynamics are much slower than in the GS, and usually in the experiments the particles stop propagating due to disorder (Fig. 1(f)). We thus cannot directly measure the small Coulombic contribution in this state; however, we can resolve it by using simulations with the same experimental conditions as in Figs. 3(a) and 3(b) and a much larger, disorder free system. The results of these simulations are shown in Fig. 3(c), where we compare the motion of single $\left(Q_{M}=0\right)$ 

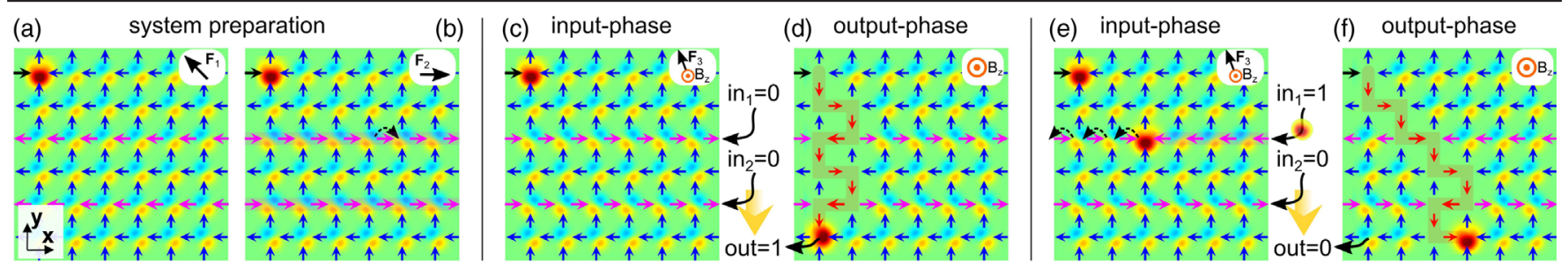

FIG. 4. Realization of a NOR gate via numerical simulation. (a),(b) Images showing the system preparation: (a) a force $F_{1}$ applied along the diagonal biases the system, except for one fixed spin; (b) a smaller force $F_{2}$ shifts two rows of particles (magenta) with high magnetic susceptibilities. (c),(d) Images showing a 1 output obtained from $(0,0)$ inputs. In (c) an external field $B_{z}$ is applied perpendicular to the plane to start defect propagation, while a small force $F_{3}$ along the diagonal prevents motion of other defects from the upper left corner. The final result shown in (d) is the 1 output. (e),(f) Images showing a 0 output obtained from $(1,0)$ input. In (e) an input current causes the whole line of magenta spin to flip (1 input). The 0 output results from the changed trajectory of the propagating defect (f). All the corresponding videos can be found in Ref. [34].

and double defects $\left(Q_{M}>0\right)$. Both have the same linetension contribution and therefore move at an identical speed for large distances. However, the closing line speeds up when the two defects are approaching at the end of the process due to sole Coulombic interaction. This time difference is shown in the inset of Fig. 3(c), and can be well fitted by Eq. (1) with $\kappa=0$ (continuous line), resulting in a similar value for the topological Coulomb charge as in the GS.

A major driving interest in studying defect dynamics in ASI lays on the possibility of realizing dissipation-free "magnetronic" circuitry $[10,42]$. We demonstrate that the colloidal spin ice system can be used to perform logic operations based on the motion of topological monopole defects. Figure 4 shows the realization, by numerical simulation, of a NOR gate, which is a functionally complete port capable of generating all logical functions [43]. The gate is completely resettable, since it requires only external fields or gradients to work, and not individual manipulation via laser tweezers. It is realized in a biased system, which could be formed and reset by an external magnetic force $\boldsymbol{F} \sim(\boldsymbol{B} \cdot \nabla \boldsymbol{B})$ applied along one diagonal direction, $\boldsymbol{F}_{1}=F_{1}(\hat{\boldsymbol{y}}-\hat{\boldsymbol{x}})$. In the preparation step [Fig. 4(a)] the system is biased by a force $F_{1}=2.8 \mathrm{pN}$, which displaces all particles except for a pinned one which represents a fixed spin, [top left corner in Fig. 4(a)]. We use a second type of paramagnetic colloids with a higher magnetic susceptibility, $\chi_{2}$ and ratio $\chi_{2} / \chi_{1}=1.15$, a prerequisite which forced us to restrict the realization only to the numerical scheme. These particles are placed along two parallel rows spaced by two lattice constants (magenta arrows in Fig. 4). In the second preparation step [Fig. 4(b)] these particles are selectively manipulated by a small in-plane force $\boldsymbol{F}_{2}=F_{2} \hat{\boldsymbol{x}}, F_{2}=1.6 \mathrm{pN}$, while all other particles $\left(\chi_{1}\right)$ remain at rest. The two rows represent the inputs of the logic gate: a 0 (1) is associated with a shifted (unshifted) row. After preparation of the system, a $B_{z}=$ $15 \mathrm{mT}$ field perpendicular to the plane induces the defect propagation, Figs. 4(c)-4(f). The output of the gate is measured at the bottom left corner of the sample: it is 1 if there is a magnetic current, 0 otherwise. Figures $4(\mathrm{c})-4(\mathrm{~d})$ describe the situation of the input $(0,0)$ with output 1 , while Figs. 4(e)-4(f) have input $(1,0)$ and output 0 . In the third step [Figs. 4(c) and 4(e)] a small locking force $\boldsymbol{F}_{3}=F_{3}(\hat{\boldsymbol{y}}-\hat{\boldsymbol{x}}), F_{3}=0.7 \mathrm{pN}$ is applied to hold the defect in place while the input is prepared. Now let us consider the case of the $(1,0)$ input: an applied magnetic current causes the upper first magenta line to flip back into the $x<0$ direction. In Fig. 4(f), $F_{3}$ is set to 0 and the defect starts moving. When it reaches the flipped input row, the defect changes its path, ending in a different place; thus the output is 0 . Since only $(0,0)$ input gives a 1 output, our logic port behaves like a NOR gate. A similar system could be engineered in nanoscale ASI using islands of different size or magnetic materials, which would give spins that behave differently under an external field. In this context, a recent work demonstrated the possibility to reorient the magnetization of the nanoislands in ASI with an MFM tip [44].

In summary, we studied the defect dynamics in an artificial colloidal spin ice in the GS and in the biased state and directly measure their energetic contributions. Our findings also confirm former theoretical assumptions and clearly demonstrate that these defects behave like bound magnetic monopoles. We finally demonstrate a resettable functionally complete NOR gate. The possibility to control topological monople defects in spin ice states may foster the realization of novel memory and logic devices based on magnetic current $[42,45,46]$.

We thank Andras Libal and Demian Levis for stimulating discussions. This work was supported by the ERC StG No. 335040. P. T. acknowledges support from Mineco (Project No. FIS2013-41144-P) and AGAUR (Project No. 2014SGR878).

*ptierno@ub.edu

[1] S. T. Bramwell and M. J. P. Gingras, Science 294, 1495 (2001).

[2] N. Choudhury, L. Walizer, S. Lisenkov, and L. Bellaiche, Nature (London) 470, 513 (2011). 
[3] K. Kim, M.-S. Chang, S. Korenblit, R. Islam, E. E. Edwards, J. K. Freericks, G.-D. Lin, L.-M. Duan, and C. Monroe, Nature (London) 465, 590 (2010).

[4] Y. Han, Y. Shokef, A. M. Alsayed, P. Yunker, T. C. Lubensky, and A. G. Yodh, Nature (London) 456, 898 (2008).

[5] D. Bryngelson and P. G. Wolynes, Proc. Natl. Acad. Sci. U.S.A. 84, 7524 (1987).

[6] M. J. Harris, S. T. Bramwell, D. F. McMorrow, T. Zeiske, and K. W. Godfrey, Phys. Rev. Lett. 79, 2554 (1997).

[7] A. P. Ramirez, A. Hayashi, R. J. R. J. Cava, R. B. Siddharthan, and S. Shastry, Nature (London) 399, 333 (1999).

[8] R. Moessner and A. P. Ramirez, Phys. Today 59, 24 (2006).

[9] R. F. Wang, C. Nisoli, R. S. Freitas, J. Li, W. McConville, B. J. Cooley, M. S. Lund, N. Samarth, C. Leighton, V. H. Crespi, and P. Schiffer, Nature (London) 439, 303 (2006).

[10] C. Nisoli, R. Moessner, and P. Schiffer, Rev. Mod. Phys. 85, 1473 (2013).

[11] S. A. Daunheimer, O. Petrova, O. Tchernyshyov, and J. Cumings, Phys. Rev. Lett. 107, 167201 (2011).

[12] Z. Budrikis, J. P. Morgan, J. Akerman, A. Stein, P. Politi, S. Langridge, C. H. Marrows, and R. L. Stamps, Phys. Rev. Lett. 109, 037203 (2012).

[13] U. B. Arnalds, A. Farhan, R. V. Chopdekar, V. B. Kapaklis, A. Balan, E. T. Papaioannou, M. Ahlberg, N. Frithjof, L. J. Heyderman, and B. Hjörvarsson, Appl. Phys. Lett. 101, 112404 (2012).

[14] S. Zhang, I. Gilbert, C. Nisoli, G.-W. Chern, M. J. Erickson, L. O. Brien, C. Leighton, P. E. Lammert, V. H. Crespi, and P. Schiffer, Nature (London) 500, 553 (2013).

[15] A. Farhan, P. M. Derlet, A. Kleibert, A. Balan, R. V. Chopdekar, M. Wyss, J. Perron, A. Scholl, F. Nolting, and L. J. Heyderman, Phys. Rev. Lett. 111, 057204 (2013).

[16] S. Ladak, D. E. Read, G. K. Perkins, L. F. Cohen, and W. R. Branford, Nat. Phys. 6, 359 (2010).

[17] J. P. Morgan, A. Stein, S. Langridge, and C. H. Marrows, Nat. Phys. 7, 75 (2011).

[18] E. Mengotti, L. J. Heyderman, A. F. Rodríguez, F. Nolting, R. V. Hügli, and H.-B. Braun, Nat. Phys. 7, 68 (2011).

[19] C. Phatak, A. K. Petford-Long, O. Heinonen, M. Tanase, and M. De Graef, Phys. Rev. B 83, 174431 (2011).

[20] V. Kapaklis, U. B. Arnalds, A. Farhan, R. V. Chopdekar, A. Balan, A. Scholl, L. J. Heyderman, and B. Hjörvarsson, Nat. Nanotechnol. 9, 514 (2014).

[21] L. A. Mól, R. L. Silva, R. C. Silva, A. R. Pereira, W. A. Moura-Melo, and B. V. Costa, J. Appl. Phys. 106, 063913 (2009).

[22] L. A. S. Mól, W. A. Moura-Melo, and A. R. Pereira, Phys. Rev. B 82, 054434 (2010).

[23] R. C. Silva, F. S. Nascimento, L. A. Mól, W. A. Moura-Melo, and A. R. Pereira, New J. Phys. 14, 015008 (2012).

[24] F. S. Nascimento, L. A. Mól, W. A. Moura-Melo, and A. R. Pereira, New J. Phys. 14, 115019 (2012).

[25] R. C. Silva, R. J. C. Lopes, L. A. S. Mól, W. A. MouraMelo, G. M. Wysin, and A. R. Pereira, Phys. Rev. B 87, 014414 (2013).
[26] D. Levis and L.F. Cugliandolo, Phys. Rev. B 87, 214302 (2013).

[27] E. Y. Vedmedenko, Phys. Rev. Lett. 116, 077202 (2016).

[28] V. S. Bhat, F. Heimbach, I. Stasinopoulos, and D. Grundler, Phys. Rev. B 93, 140401 (2016).

[29] S. T. Bramwell, S. R. Giblin, S. Calder, R. Aldus, D. Prabhakaran, and T. Fennell, Nature (London) 461, 956 (2009).

[30] L. D. C. Jaubert and P. C. W. Holdsworth, J. Phys. Condens. Matter 23, 164222 (2011).

[31] C. Castelnovo, R. Moessner, and S. Sondhi, Nature (London) 451, 42 (2008).

[32] A. Libál, C. Reichhardt, and C. J. Olson Reichhardt, Phys. Rev. Lett. 97, 228302 (2006).

[33] A. Libál, C. Reichhardt, and C. J. Olson Reichhardt, Phys. Rev. E 86, 021406 (2012).

[34] See Supplemental Material at http://link.aps.org/ supplemental/10.1103/PhysRevLett.117.168001 for more details on the experimental system, the numerical simulation, and seven video clips illustrating the defect dynamics, which includes Refs. [35,36].

[35] A. Ortiz-Ambriz and P. Tierno, Nat. Commun. 7, 10575 (2016).

[36] K. Jacobs, Stochastic Processes for Physicists: Understanding Noisy Systems (Cambridge University Press, Cambridge, England, 2010), ISBN .

[37] To calculate the gravitational potentials we use the density mismatch $\Delta \rho=0.9 \mathrm{gcm}^{-3}$ between the particle and the suspending medium. Optical tweezers are used to load one particle per double well, to order the system or create defects.

[38] C. Nisoli, New J. Phys. 16, 113049 (2014).

[39] The small system size was chosen in order to both make more evident the effect of Coulombic-like interactions, and to minimize the breaking of the defect line caused by small disorder related with the distribution of hills, Fig. 1(f).

[40] The friction between such particles is given by $\gamma=6 \pi \eta d / 2$, being $\eta=10^{-3} \mathrm{~Pa} \cdot \mathrm{s}$ the viscosity of the medium (water).

[41] It is possible to create a single topological monopole which propagates in the bias state purely due to line tension because of the finite system size. Once a charged defect is created, an oppositely charged one is needed in order to fulfill charge conservation. The flipped spins simply place one of the two defects outside the border region, such that it does not interact with the system.

[42] S. R. Giblin, S. T. Bramwell, P. C. W. Holdsworth, D. Prabhakaran, and I. Terry, Nat. Phys. 7, 252 (2011).

[43] T. C. Bartee, Computer Architecture and Logic Design (McGraw-Hill, New York, 1991).

[44] Y.-L. Wang, Z.-L. Xiao, A. Snezhko, J. Xu, L. E. Ocola, R. Divan, J. E. Pearson, G. W. Crabtree, and W.-K. Kwok, Science 352, 962 (2016).

[45] S. J. Blundell, Phys. Rev. Lett. 108, 147601 (2012).

[46] I. Gilbert, G.-W. Chern, B. Fore, Y. Lao, S. Zhang, C. Nisoli, and P. Schiffer, Phys. Rev. B 92, 104417 (2015). 